\title{
Covert Channels in Multiple Access Protocols
}

\author{
Seyed Ali Ahmadzadeh \\ University of Waterloo, Canada \\ ahmadzdh@uwaterloo.ca
}

\author{
Gordon B. Agnew \\ University of Waterloo, Canada \\ gbagnew@uwaterloo.ca
}

\begin{abstract}
In this paper, the use of structural behavior of communication protocols in designing new covert channels is investigated. In this way, a new covert transmitter is designed based on a modified CSMA protocol that enables the transmitter to embed a covert message in its overt traffic. The proposed scheme provides high covert rate without compromising the stealthiness of the channel.
\end{abstract}

\section{Categories and Subject Descriptors}

C.2.1 [Computer-Communication Networks]: Network Architecture and Design-Wireless communication

\section{General Terms}

Algorithms, Design, Security

\section{INTRODUCTION}

Covert communication often refers to the process of communicating through a channel that is neither designed, nor intended to transfer data [1]. Covert channels may be used to allow information to be leaked to an unauthorized recipient by exploiting weaknesses in conventional communication systems. Kemmer [1] identified three necessary conditions for existence of a covert channel. (i) a global resource that is shared between the transmitter and the receiver, (ii) ability to modify the shared resource, and (iii) a method to achieve synchronization between the transmitter and the receiver.

The wireless channel provides all three conditions making it a perfect medium for a covert channel. In [2] a covert channel based on jamming over slotted ALOHA was introduced. Later, a covert channel that exploits the properties of splitting tree collision resolution algorithm was proposed in [3]. Wang et al. extended the aforementioned approach into an anonymous covert channel [4] in which the receiver decodes the message using a voting approach that considers the probabilistic decisions of multiple covert transmitters.

Although the above schemes provide stealth covert channels, they trade the achievable covert rate in favor of the channel secrecy. Moreover, in order to avoid detection, these schemes are designed based on keeping the covert transmitter's long-term statistical characteristics as close as possible to an ordinary transmitter. However, to achieve this goal, the transmitter has to deviate from short-term behaviors of a regular source which may be used by a system observer to uncover the existence of the covert channel.

Copyright is held by the author/owner(s).

SIGCOMM'11, August 15-19, 2011, Toronto, Ontario, Canada.

ACM 978-1-4503-0797-0/11/08.

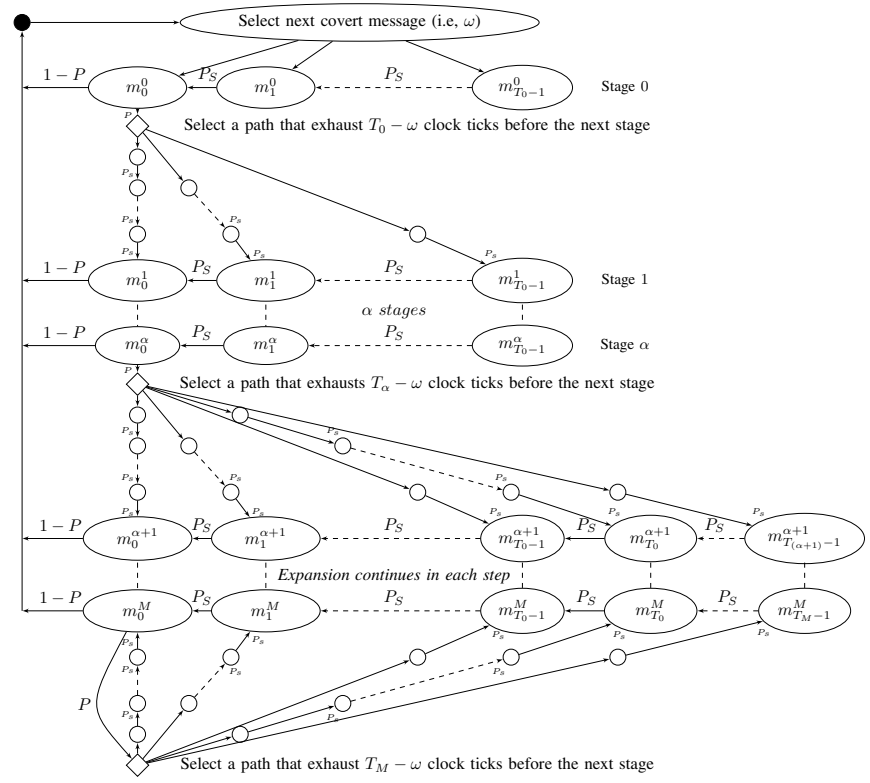

Figure 1: Covert message transmission. $P_{S}$ is the probability of a successful transmission by members of the covert set.

In this paper, we present a new approach that systematically exploits the probabilistic nature of multiple access protocols in order to open a covert channel in the system. To this end, we turn our attention to the carrier sense multiple access (CSMA) protocol and design a covert transmitter that mimics the characteristics of a regular CSMA node while communicates covertly with the covert receiver.

\section{COVERT CHANNEL DESIGN}

In principle, the proposed covert channel benefits from the channel activities of a subset of users in the system (i.e., the covert set) as the mean of synchronization, and the covert transmitter's overt traffic is used in order to modify the shared medium. The design is based on a modified version of the CSMA protocol that gives the transmitter enough freedom to embed the covert message into its overt traffic, yet the transmitter reacts to the network events (e.g., collision) similar to an ordinary CSMA node.

Figure 1 depicts the modified CSMA protocol which is used by the covert transmitter. The transmitter and the receiver are equipped with a virtual clock called covert clock. The covert clock is incremented every time a packet from members of the covert set is detected. Due to the broadcast nature of the wireless channel, the transmitter and the receiver can observe the channel activities of the members of the covert set and increment their clock synchronously. This only requires that both sides track the same set of users and 
Table 1: Details of Simulation Scenarios

\begin{tabular}{|c|c|c|c|c|c|c|}
\hline \multicolumn{3}{|c|}{ Parameter } & $\mathrm{SC} 1$ & $\mathrm{SC} 2$ & SC3 & $\mathrm{SC} 4$ \\
\hline \multicolumn{3}{|c|}{ Number of users $(N)$} & 25 & 35 & 50 & 15 \\
\hline \multicolumn{3}{|c|}{ Size of the covert set $(|S|)$} & 16 & 21 & 33 & 10 \\
\hline \multicolumn{3}{|c|}{ Covert transmitter min window size $\left(T_{0}\right)$} & 4 & 7 & 8 & 4 \\
\hline \multicolumn{3}{|c|}{ Expansion postpone parameter $(\alpha)$} & 1 & 1 & 1 & 1 \\
\hline \multicolumn{3}{|c|}{ Regular user min window size $\left(W_{\min }\right)$} & 16 & 32 & 32 & 16 \\
\hline \multicolumn{3}{|c|}{ Number of back off stages $(M)$} & 6 & 5 & 5 & 6 \\
\hline \multicolumn{7}{|c|}{ Table 2: Security Tests for Different Scenarios } \\
\hline Scenario & KS-test & $\begin{array}{c}\text { Regularity Score } \\
\text { (Covert Transmitter) } \\
\end{array}$ & \multicolumn{4}{|c|}{$\begin{array}{c}\text { Regularity Score } \\
\text { (Regular Transmitter) }\end{array}$} \\
\hline $\mathrm{SC} 1$ & 0.0432 & 0.2704 & \multicolumn{4}{|c|}{0.2757} \\
\hline $\mathrm{SC} 2$ & 0.0398 & 0.2322 & \multicolumn{4}{|c|}{0.2509} \\
\hline $\mathrm{SC} 3$ & 0.0367 & 0.3772 & \multicolumn{4}{|c|}{0.3794} \\
\hline $\mathrm{SC} 4$ & 0.0353 & 0.2327 & \multicolumn{4}{|c|}{0.2534} \\
\hline
\end{tabular}

are equipped with proper error correction methods in case a mismatch happens between the transmitter and the receiver.

Each covert message (i.e., $\omega$ ) is associated to a unique state in the first stage of the transmitter's transmission window (i.e., stage 0). The covert communication begins as the transmitter moves to the corresponding state of the covert message. Then, the transmitter monitors the channel to catch packets from members of the covert set. For each packet, the transmitter's clock is incremented by one unit and it moves down one state in its transmission window (to the left in Figure 1). The transmitter sends its next packet when it reaches the last state of the transmission window.

The receiver also maintains its covert clock similar to the transmitter. Hence, upon receiving a packet from the transmitter, the receiver reads the value of its clock, decodes the covert message, and resets the clock for the next message.

However, if the transmitter fails to transmit the packet on the proper time slot (e.g., due to collision), it expands its contention window and selects a new time slot that corresponds to the covert message. Indeed, this window expansion plays a critical role to maximize the stealthiness of the covert transmitter as it mimics the behavior of an ordinary CSMA node to handle collisions in the network. Thus,

$$
T_{i}= \begin{cases}T_{0} & 0 \leq i \leq \alpha \\ 2 \times T_{i-1} & \alpha<i \leq M \\ T_{M} & i>M\end{cases}
$$

Where, $T_{i}$ is the size of the transmitter's contention window in the $i^{\text {th }}$ stage, and $M$ is the number of backoff stages. The parameter $\alpha$, is a design parameter that controls how far the transmitter deviates from behaviors of a regular user.

Finally, in order to keep synchronization between the transmitter and the receiver, following each unsuccessful packet transmission attempt, the transmitter waits for $T_{i}-\omega$ extra clock ticks (i.e., packets from members of the covert set) before moving to the next stage. Hence, at the beginning of the $i^{\text {th }}$ stage, the covert clocks at the receiver and the transmitter would be equal to $\sum_{j=0}^{i-1} T_{j}$, regardless of the covert message. The receiver removes this offset from its covert clock (i.e., $C_{r}$ ) and decodes the message as: $\omega=C_{r} \bmod T_{0}$.

\section{PERFORMANCE ANALYSIS RESULTS}

The performance analysis is performed on four scenarios (Table 1) using a CSMA testbed with Slot time $=20 \mu \mathrm{s}$, $\mathrm{SIFS}=10 \mu s, \mathrm{DIFS}=50 \mu s$, Payload $=1.5 \mathrm{~KB}$, and Channel overt rate $=1 \mathrm{Mbps}$.

In order to evaluate the stealthiness of the channel, we use the Kolmogorov-Smirnov test (KS-test) [5] and the reg-

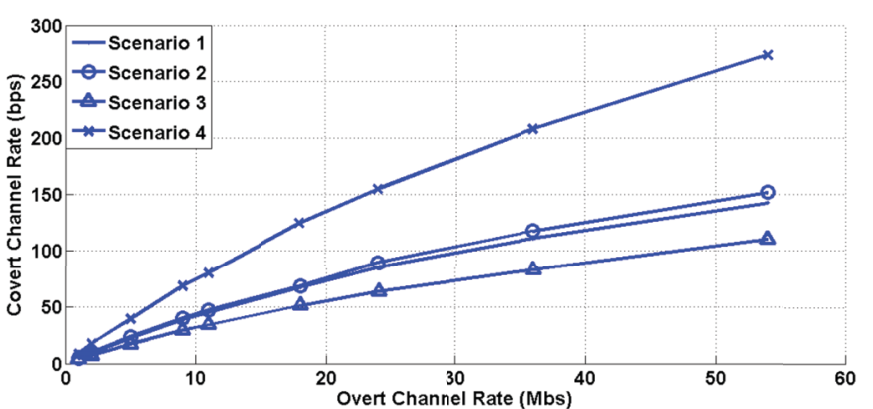

Figure 2: Covert rate of the proposed channel.

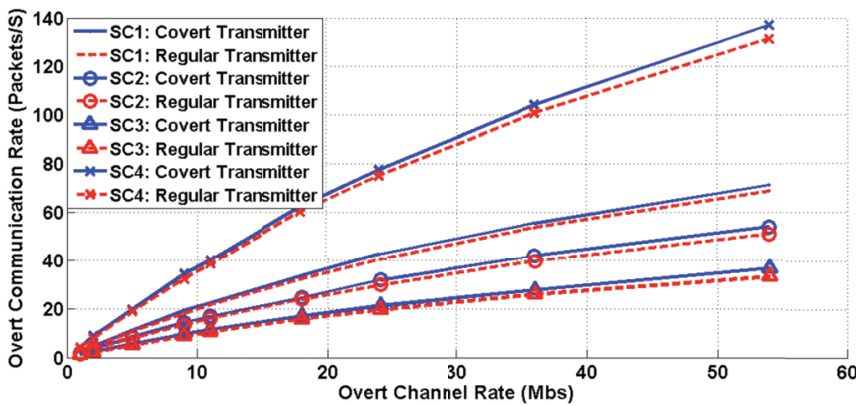

Figure 3: Overt rate of the covert transmitter

ularity test [6]. The KS-test shows the difference between the distributions of the inter-packet delays sampled from the covert transmitter's traffic and the traffic originated from a regular node. The regularity test is designed to detect the abnormal behavior of the covert transmitter in reacting to the network events (e.g., packet loss). The test results in Table 2 verifies that the transmitter has similar long-term characteristics (KS-test) and short-term behaviors (regularity test) as compared to a regular CSMA node.

Figure 2 shows the achievable rate of the proposed covert channel. It is noted that the covert channel rate increases linearly with the capacity of the overt channel. The overt communication rate of the covert transmitter and regular users in the system are depicted in Figure 3. From the graph, it can be observed that the transmitter conveys the same overt rate as compared to regular users of the system. Thus, it is extremely difficult for a system observer to track the transmitter based on overt communication rate.

\section{REFERENCES}

[1] R. Kemmerer. Shared resource matrix methodology: An approach to identifying storage and timing channels. ACM Trans. on Computer Systems, 1(3):277, 1983.

[2] S. Bhadra, S. Bodas, S. Shakkottai, and S. Vishwanath. Communication Through Jamming Over a Slotted ALOHA Channel. IEEE Trans. on Information Theory, pages 54(11):5257, 2008.

[3] S. Li and A. Ephremides. A covert channel in MAC protocols based on splitting algorithms. In IEEE $W C N C$, pages 1168-1173, 2005.

[4] Z. Wang, J. Deng, R. Lee, and P. Princeton. Mutual anonymous communications: a new covert channel based on splitting tree MAC. In IEEE INFOCOM, pages 2531-2535, 2007.

[5] Y. Liu, D. Ghosal, F. Armknecht, A. Sadeghi, S. Schulz, and S. Katzenbeisser. Hide and Seek in Time: Robust Covert Timing Channels. In ESORICS, pages 120-135, 2010.

[6] S. Cabuk, C. Brodley, and C. Shields. IP covert timing channels: design and detection. In $A C M C C S$, pages 178-187, 2004. 